\title{
Transapical sutureless aortic valve implantation under magnetic resonance imaging guidance: Acute and short-term results
}

\author{
Keith A. Horvath, MD, Dumitru Mazilu, PhD, Junfeng Cai, MD, PhD, Bogdan Kindzelski, BS, and
} Ming Li, PhD

\begin{abstract}
Objectives: Despite the increasing success and applicability of transcatheter aortic valve replacement, 2 critical issues remain: the durability of the valves, and the ideal imaging to aid implantation. This study was designed to investigate the transapical implantation of a device of known durability using real-time magnetic resonance imaging (MRI) guidance.
\end{abstract}

Methods: A sutureless aortic valve was used that employs a self-expanding nitinol stent and is amenable to transapical delivery. MRI (1.5-T) was used to identify the anatomic landmarks in 60-kg Yucatan swine. Prostheses were loaded into an MRI-compatible delivery device with an active guidewire to enhance visualization. A series of acute feasibility experiments were conducted $(\mathrm{n}=10)$. Additional animals $(\mathrm{n}=6)$ were allowed to survive and had follow-up MRI scans and echocardiography at 90 days postoperatively. Postmortem gross examination was performed.

Results: The valve was MRI compatible and created no significant MRI artifacts. The 3 commissural struts were visible on short-axis view; therefore, coronary ostia obstruction was easily avoided. The average implantation time was 65 seconds. Final results demonstrated stability of the implants with preservation of myocardial perfusion and function over 90 days: the ejection fraction was $48 \% \pm 15 \%$; the peak gradient was $17.3 \pm 11.3 \mathrm{~mm} \mathrm{Hg}$; the mean gradient was $9.8 \pm 7.2 \mathrm{~mm} \mathrm{Hg}$. Mild aortic regurgitation was seen in 4 cases, trace in 1 case, and a severe central jet in 1 case. Prosthesis positioning was evaluated during gross examination.

Conclusions: We demonstrated that a sutureless aortic valve can be safely and expeditiously implanted through a transapical approach under real-time MRI guidance. Postimplantation results showed a well-functioning prosthesis, with minimal regurgitation, and stability over time. (J Thorac Cardiovasc Surg 2015;149:1067-72)

See related commentary on pages 1072-3.

Surgical aortic valve replacement is the standard treatment for patients with aortic stenosis. Recently, transcatheter aortic valve replacement (TAVR) has been considered as an alternative treatment to reduce mortality in patients who are at high risk or are considered inoperable. ${ }^{1-3}$ This intervention utilizes bioprosthetic valves, which are delivered and implanted within the diseased aortic valve through catheters, using either a transarterial or transapical approach. ${ }^{4,5}$

Fluoroscopy and transesophageal echocardiography as imaging modalities for TAVR have limitations, including

\footnotetext{
From the Cardiothoracic Surgery Research Program, National Heart, Lung, and Blood Institute, National Institutes of Health, Bethesda, Md.

Funded by the NHLBI Division of Intramural Research.

Disclosures: Authors have nothing to disclose with regard to commercial support.

Received for publication Aug 17, 2014; revisions received Oct 16, 2014; accepted for publication Oct 26, 2014; available ahead of print Nov 25, 2014.

Address for reprints: Keith A. Horvath, MD, Cardiothoracic Surgery Research Program, National Heart, Lung, and Blood Institute, National Institutes of Health, Building 10, Room B1D47, 10 Center Drive, Bethesda, MD 20892 (E-mail: horvathka@mail.nih.gov).

$0022-5223 / \$ 36.00$

Copyright (C) 2015 by The American Association for Thoracic Surgery

http://dx.doi.org/10.1016/j.jtcvs.2014.10.101
}

poor anatomic visualization and lack of soft-tissue contrast; landmark loss; a requirement for rapid ventricular pacing; intravenous contrast toxicity; and substantial radiation exposure to both the patient and the operative team. ${ }^{6,7}$ Magnetic resonance imaging (MRI) offers an alternative means of imaging and overcomes many of the limitations of fluoroscopy. Specifically, MRI provides excellent anatomic visualization, particularly in its ability to provide high-resolution images of blood-filled structures. Vascular, as well as soft-tissue, visualization can easily be performed simultaneously with MRI. The development of real-time MRI (rtMRI) allows this imaging modality to provide intraoperative guidance for delivery of prosthetic aortic valves. Moreover, MRI-guided surgery allows direct functional assessments to be made before, during, and immediately after an intervention that are not obtainable by conventional imaging alone.

Our group has successfully performed transapical aortic valve replacements using rtMRI guidance. ${ }^{8,9}$ We have reported the implantation of both balloon-expandable and self-expanding prostheses with midterm follow-up. ${ }^{10,11}$ Our experience showed that rtMRI-guided TAVR is a novel and reproducible method that achieves accurate positioning of the bioprosthesis.

The Perceval S valve (Sorin Group, Saluggia, Italy) is CE (Conformité Européene) mark approved and is indicated for 


\section{Abbreviations and Acronyms \\ MRI = magnetic resonance imaging \\ rtMRI $=$ real-time MRI \\ TAVR $=$ transcatheter aortic valve replacement}

both standard and minimally invasive surgical replacement of the aortic valve in patients suffering from aortic stenosis and aortic insufficiency. The valve's functional component is made of bovine pericardium and is mounted on a super-elastic nitinol alloy frame. The self-anchoring configuration eliminates the need to suture the prosthesis into place, thereby reducing procedure time for aortic valve replacement. The preliminary results of a European multicenter pilot trial confirmed the safety and efficacy of the valve in a high-risk cohort of patients. ${ }^{12}$ Use of this valve for sutureless aortic valve replacement showed outcomes in propensity-scored analysis that are similar to those in sutured surgical aortic valve replacement. ${ }^{13}$ The nitinol alloy frame displays no significant artifacts in MRI, and its self-expanding mechanism allows the valve to be delivered through a transcatheter approach.

In this study, we sought to prove the safety and efficiency of rtMRI guidance for TAVR, and test the hypothesis that a sutureless aortic valve can be properly implanted under rtMRI guidance. We report the preclinical feasibility of valve implantation via an off-label transapical approach under rtMRI guidance, including acute feasibility and 90-day results.

\section{MATERIALS AND METHODS \\ Delivery Device}

A device designed for delivery of self-expanding bioprosthetic aortic valves $^{14}$ was created for deploying the Perceval S valve via the transapical approach. As described elsewhere,${ }^{14}$ the delivery device is made of plastic materials and is fully compatible with MR. The computer-aided design drawing of the delivery device is shown in Figure 1. Pushing the inner rod will advance the crimped prosthesis out of the sheath for deployment. To allow for hemostatic passage of a central guidewire, a modified CheckFlo Introducer Set (French size 14) (Cook, Bloomington, Ind) is used (5). To improve visibility of the device during the rtMRI-guided procedure, a loop coil antenna (6) was embedded into the exterior tube of the delivery system. An additional wire insulator (7) is used to protect the antenna wire. The antenna (6) is then connected to the matching/decoupling circuit box by a radio frequency connector (8).

The entire delivery system can be inserted through a 5-12 mm VersaStep Plus trocar (Tyco Healthcare Group LP, North Haven, Conn). The valve was crimped and placed inside the outer sheath at the distal end of the delivery system (Figure 2). Upon release of the valve by retraction of the outer sheath, the prosthesis expands to its original dimensions.

\section{Procedure}

All experiments were performed by a single operator under protocols approved by the National Institutes of Health Animal Care and Use Committee. A total of 16 Yucatan swine (weight, 60-65 kg) were used10 for the acute feasibility study, and 6 for the survival protocol. With the use of general endotracheal anesthesia, the animals underwent a preprocedure MRI scan. A 1.5-T MRI system (MAGNETOM Aera; Siemens Medical Solutions, Munich, Germany) was used to identify the anatomic landmarks of the aortic annulus, the coronary artery ostia, and the mitral valve leaflets. Based on the aortic annulus, the sino-tubular junction diameter, and the aortic root height, an appropriately sized valve was selected. The valve was crimped and loaded into the delivery device. One of the valve commissures was aligned with the active guide wire (MR loop coil antenna) that was embedded in the delivery device.

Interactive Front End navigation software (Siemens Corporate Research, Munich, Germany), along with an interactive real-time pulse sequence (BEAT_IRTTT), was used as real-time navigation for valve deployment. Via a small subxyphoid incision, the pericardium was opened, and the apex of the heart was exposed. Two concentric purse-string sutures were placed around the apex, through which the12-mm trocar was inserted into the left ventricle to create direct access to the aortic valve. The animals then underwent rtMRI-guided transapical aortic valve replacement without unloading by rapid ventricular pacing or cardiopulmonary bypass.

During the procedure, the animals were heparinized and monitored with electrocardiography, oxygen saturation, end-tidal $\mathrm{CO}_{2}$, systemic and left ventricular blood pressure, and arterial blood gas analysis. We recorded the time of the procedures and observed any interference of the devices with the surrounding environment during the procedures.

After placement of the valve, the trocar was removed and the apex closed via the purse-string sutures. Postplacement images were acquired to confirm the correct positioning of the prostheses, as well as the valvular and heart function. Gated cine MRI was used to assess mitral valve function and myocardial function. Phase-contrast cine MRI was used to identify flow through the new valve, as well as intravalvular or paravalvular regurgitation. An MR first-pass perfusion scan was performed during intravenous injection of gadolinium-diethylene triamine pentacetic acid (Gd-DTPA) contrast agent to confirm that myocardial blood flow was intact to all segments of the myocardium.

A series of short-term feasibility experiments were conducted $(n=10)$, in which the animals were sacrificed after valve placement and MRI assessment. Six additional animals were allowed to survive for short-term follow-up. At 1 month postoperatively, follow-up MRI scans and transthoracic echocardiograms were acquired; at 3 months postoperatively, MRI scans and confirmatory 2- and 3-dimensional transesophageal echocardiograms were acquired. Gated cine MRI, phase-contrast cine MRI, and MR first-pass perfusion scanning during intravenous injection of the contrast agent were repeated at those time points to confirm the position of the prostheses, and valvular and heart function.

\section{RESULTS}

The valve is MRI safe and compatible. It appears as a dark cylindrical cage in the MRI images, without significant artifacts (Figure 3). The 3, thick, commissural struts are visible as dark dots in the short-axis view. The dark cylindrical cage image was used to determine correct positioning and whether the valve had migrated over time; the dark commissural dots were used to determine whether the prosthesis was fully deployed and properly oriented.

The valve can be crimped and easily loaded into the delivery device (Figure 2). The average time that elapsed from crimping to loading of the valve onto the delivery device to deployment was approximately 6 minutes. In the acute feasibility study, 5 animals had an improperly deployed valve, as confirmed by gross examination. We noticed that in these animals, the anatomy of the ascending aorta was not ideal, most likely causing the stent to remain 


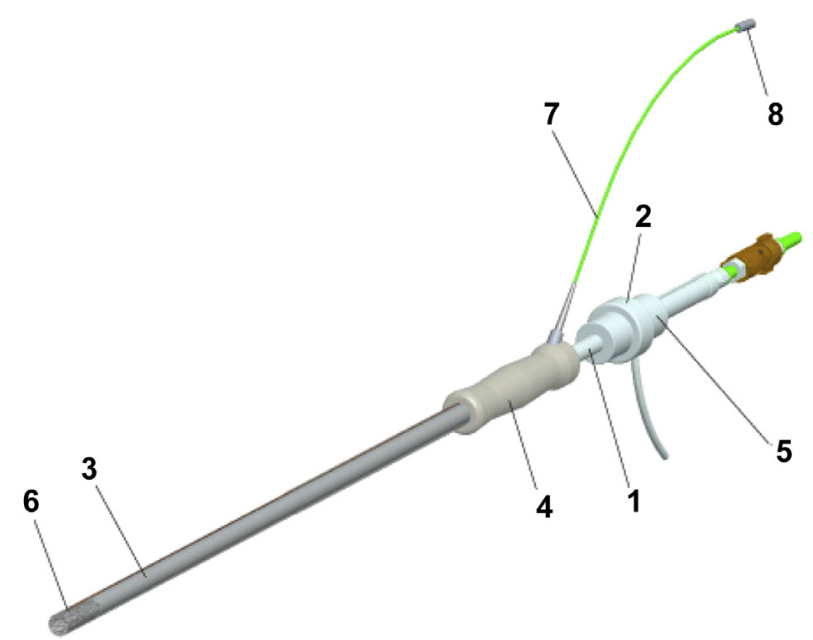

FIGURE 1. Computer-aided design drawing of the delivery device, showing a dual-lumen, coaxial tube structure containing an inner carrier rod (1), which connects to the back handgrip (2), and a coaxial outer sheath (3), which also connects to the back handgrip (4). (5) Modified Check-Flo Introducer Set (French size 14) (Cook, Bloomington, Ind). A loop coil antenna (6) is embedded into the exterior tube of the delivery system to improve visibility of the device during real-time magnetic resonance imaging. An additional wire insulator (7) protects the antenna wire. A radio frequency connector $(8)$ connects the antenna to the matching/decoupling circuit box.

folded. The diameter of the ascending aorta of these 5 animals was too small for the valve, which was selected based on only the aortic annulus. If the ascending aorta is too narrow, it prevents proper expansion of the nitinol cage after deployment.

The valve was successfully implanted in the other 5 animals once the aforementioned sizing issue was addressed by considering all of the aortic-root geometry (ie, annulus size, sino-tubular junction diameter, and root height). In these animals, postplacement gated cine MRI revealed excellent myocardial function after valve implantation in both the long- and short-axis views. The perfusion results confirmed adequacy of blood flow at the tissue level, indicating proper valve positioning with respect to the coronary ostia. The phase-contrast cine MR images confirmed good systolic flow with excellent valve-leaflet opening and no obvious evidence of turbulence or diastolic regurgitant flow in 4 animals. In 1 animal, a slight paravalvular leak was observed in the phase-contrast cine MR images. Necropsy of all these animals confirmed that the deployed prosthetic valve was properly located with respect to the aortic annulus, and that the commissures did not obstruct the coronary ostia.

In 6 surviving animals, we recorded that the deployment time-the interval from introduction of the prosthesis into the trocar to full expansion of the bioprosthesis was $65 \pm 17$ (mean \pm standard deviation) seconds. The MR microcoil antenna clearly indicated the valve orientation in
MRI during the procedure. These 6 animals in the survival protocol lived to be 3 months. Hemodynamic measurements of ventricular and valvular parameters of the 6 animals, based on 3-month follow-up echocardiograms and MRI scans, are reported: the ejection fraction was $48 \% \pm 15 \%$; the peak gradient was $17.3 \pm 11.3 \mathrm{~mm}$ $\mathrm{Hg}$; and the mean gradient was $9.8 \pm 7.2 \mathrm{~mm} \mathrm{Hg}$. Mild aortic regurgitation was seen in 4 cases, and trace in 1 . A severe central jet was observed in 1 case, with concomitant left ventricular dilatation, and severe dysfunction was demonstrated by echocardiogram and MRI.

Animals were sacrificed at 3 months, and the valves were explanted. Macroscopic examination and radiographic analysis was conducted on the explanted valves. Preliminary findings on prosthesis positioning and interaction with the heart components are reported here. On radiographic examination, none of the 6 prostheses showed evidence of stent-strut fractures (Figure 4). No leaflet mineralization was observed in 5 prostheses; mild mineralization of all leaflets was visible in the remaining prostheses.

On macroscopic examination, valve positioning and orientation were evaluated (Figure 5). All of the prostheses were fully expanded in the aortic root, with proper positioning and slightly enlarged aortic diameters. On the ventricular side, no interference was observed between the prosthesis and the mitral valve. In the long-axis view, 1 prosthesis seemed to be implanted too high. The other 5 prostheses were well positioned. Three prostheses were expanded in the aortic root, slightly tilted with respect to the long axis, 1-2 mm lower at the right coronary ostium. Despite the slightly tilted positioning of the stent, both coronary ostia were patent in these 3 cases, and the valves functioned well. Gross pathology in the case of the severe central regurgitation revealed that the prosthesis was implanted too high; although it had expanded, it prevented the valve from attaining a circular shape and led to leaflet displacement. In addition, signs of pericarditis were observed in this case.

At macroscopic examination, we observed that the stent struts were mostly covered with pannus formation up to the distal crown in all 6 prostheses. In 2 prostheses, stent struts were embedded in the aortic wall, which may be a result of the narrower pig aortic-root geometry. Jet lesions and fibrous deposition on the ventricular wall in proximity to the prosthesis skirt suggest the presence of paravalvular leakage in 2 animals, consistent with the MRI and echo findings.

\section{DISCUSSION}

We demonstrated that rtMRI guidance for TAVR is safe and efficient, and the Perceval $\mathrm{S}$ valve can be implanted through a transapical approach under MRI guidance. MRI may be used for preprocedural planning, intraoperative 

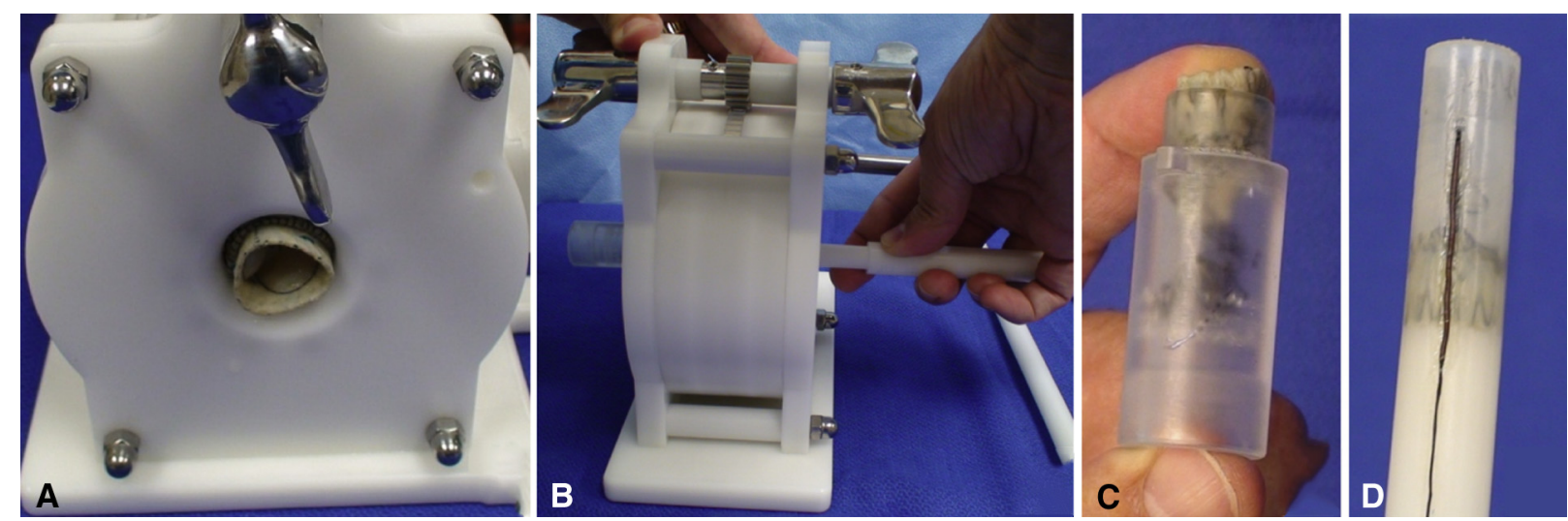

FIGURE 2. A, The valve is inserted into a homemade crimper. B, The valve is crimped and is transferred to a temporary holder. C, The temporary holder is used to transfer the crimped valve from the crimper to the delivery device. $\mathrm{D}$, The crimped valve is loaded inside a homemade delivery device.

guidance, and postinterventional assessment, all in a single hybrid operating room suite. ${ }^{15}$ Use of this rtMRI guidance may expand the utility of TAVR, targeting patients with renal insufficiency, a contraindication to the procedure. ${ }^{16,17}$ Furthermore, rtMRI allows for continuous evaluation of the delivery of the prosthesis, provides excellent views of valvular and annular anatomy, and can be performed in a beating heart without necessitating ventricular unloading. Therefore, MRI as an imaging modality may lead to improved results, including more-accurate device placement within the aortic annulus; avoidance of the risk of hemodynamic compromise, owing to the lack of need for ventricular unloading; and immediate evaluation of heart function and perfusion after valve implantation.

The Perceval $\mathrm{S}$ is a prosthetic valve comprising leaflets of bovine pericardium fixed in a stent of super-elastic alloy. The self-expanding nitinol frame is MRI safe and compatible. Postimplantation results indicate wellfunctioning prostheses, with minimal regurgitation, and good stability over time. To fully implement the transapical approach, a series of modifications of the delivery device and crimper had to be made. The method we used to deliver this valve was the same as we described elsewhere, ${ }^{10}$ which depends on the stent itself to expand by simply returning to its original shape. The design of the stent of the valve features 2 ring segments, 1 on the proximal end (the "outflow" ring), and 1 on the distal end (the "inflow" ring). Connecting elements are designed to support the valve and allow the prosthesis to anchor to the aortic root and the sinuses of Valsalva. The struts of the stent bulge outward from the middle part to fill the coronary sinuses.

Proper valve size selection was the key factor in our experimental study. The importance of proper prosthesis sizing has been shown clinically. ${ }^{18}$ For Perceval $\mathrm{S}$ valve
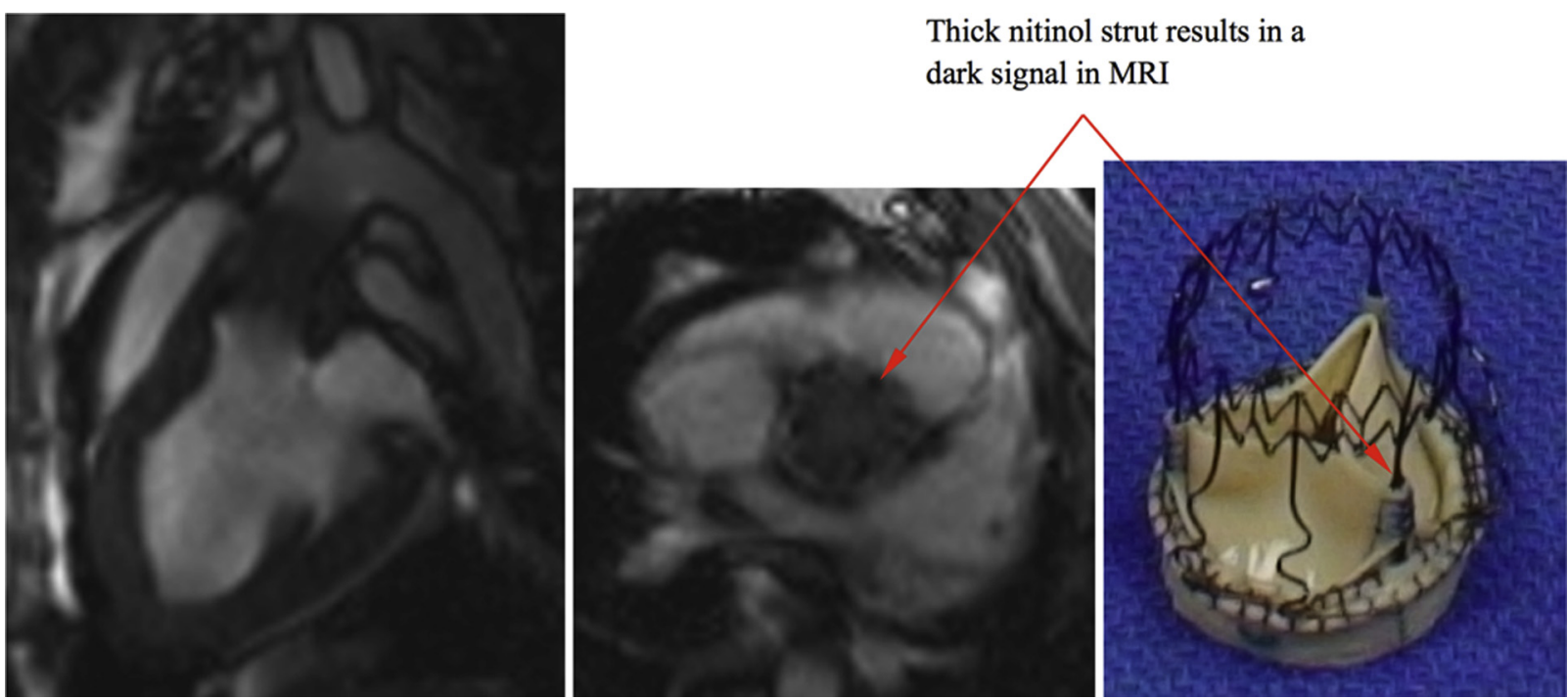

FIGURE 3. The valve appears as a dark cylindrical cage in the MRI images. MRI, Magnetic resonance imaging. 


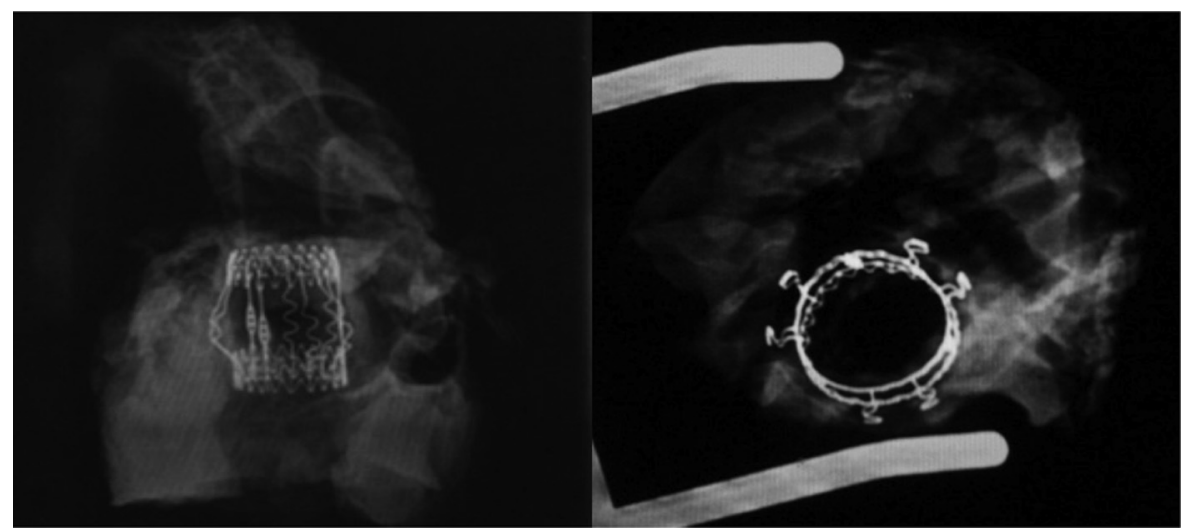

FIGURE 4. Radiographic image of the valve.

size selection, multiple anatomic characteristics-aortic annulus, sino-tubular junction diameter, and aortic-root height — need to be evaluated. The valve was selected based on only the aortic annulus, which could have caused the outflow portion of the valve to remain folded after deployment. The valve is designed for humans, and for placement in an open sutureless fashion; its outwardbowing struts are critical elements for such an application. However, we used Yucatan swine as the animal model, and the sinuses of the aortic root do not bulge out as much in pigs as they do in humans. Aside from the issue of size selection, the difference in the aortic-root geometry between swine and humans led to aortic-root enlargement in some cases.

Proper positioning of the valve was the other important factor in our experimental study. If the valve is implanted too high in the aortic root, the aortic root may prevent the prosthesis from expanding into a perfectly circular shape. A shape that is not a perfect circle can cause tenting of a leaflet, which leads to aortic regurgitation.

We found that the self-expansion of the valve can push aside the healthy native valve in our animal model. The prosthesis is designed to be implanted after surgical explant of the calcified native aortic valve. If this prosthesis were implanted into a calcified aortic valve, balloon dilation, and likely native valve removal, would be necessary before implantation.

Currently, 2 prosthetic aortic valves have been approved by the US Food and Drug Administration-SAPIEN and CoreValve. The CoreValve prosthesis (Medtronic, Minneapolis, Minn) uses a trileaflet porcine pericardial valve mounted on a self-expanding nitinol frame. It may therefore be a good candidate for implantation under rtMRI guidance. The SAPIEN heart-valve system (Edwards Lifesciences, Inc, Irvine, Calif), however, cannot be implanted under rtMRI guidance, owing to its stainless-steel stent. Future studies are warranted in exploring the use of rtMRI-guided TAVR using other commercially available prostheses, such as CoreValve, and delineating the use of this approach, including investigating the clinical feasibility in patients with renal insufficiency.

The authors thank Sorin Group for providing us with Perceval valves. The authors thank Ing. Ornella Ieropoli and Dr Piero
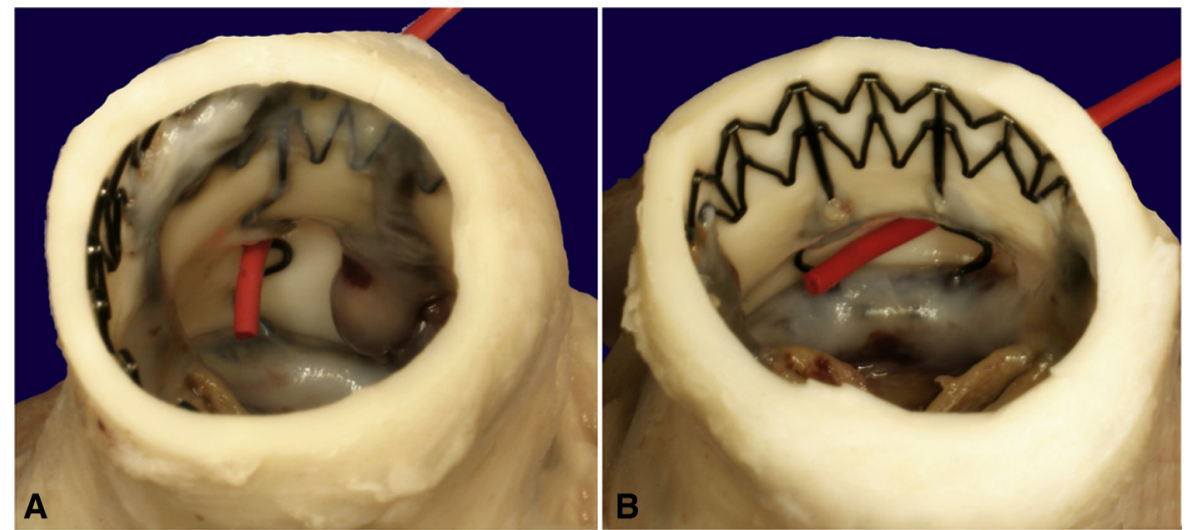

FIGURE 5. A, Right coronary ostium; (B) left coronary ostium. Both coronary ostia are at the level of the prosthesis sinusoidal struts, and they are not obstructed. 
Trabucco from Sorin Group for support with the gross examination of the explanted devices.

\section{References}

1. Leon MB, Smith CR, Mack M, Miller DC, Moses JW, Svensson LG, et al. Transcatheter aortic-valve implantation for aortic stenosis in patients who cannot undergo surgery. N Engl J Med. 2010;363:1597-607.

2. Walther T, Möllmann H, Blumenstein J, Kempfert J. Transcatheter aortic valve implantation for severe aortic stenosis-overcoming the challenges. Interv Cardiol. 2011;6:165-9.

3. Cribier A. Development of transcatheter aortic valve implantation (TAVI): a 20-year odyssey. Archiv Cardiovasc Dis. 2012;105:146.

4. Johansson M, Nozohoor S, Kimblad PO, Harnek J, Olivecrona GK, Sjögren J. Transapical versus transfemoral aortic valve implantation: a comparison of survival and safety. Ann Thorac Surg. 2011;91:57-63.

5. D’Onofrio A, Rizzoli G, Messina A, Alfieri O, Lorusso R, Salizzoni S, et al. Conventional surgery, sutureless valves, and transapical aortic valve replacement: What is the best option for patients with aortic valve stenosis? A multicenter, propensity-matched analysis. J Thorac Cardiovasc Surg. 2013; 146:1065-70.

6. Gotzmann M, Korten M, Bojara W, Lindstaedt M, Rahlmann P, Mügge A, et al. Long-term outcome of patients with moderate and severe prosthetic aortic valve regurgitation after transcatheter aortic valve implantation. Am J Cardiol. 2012; 110:1500-6.

7. Gilard M, Eltchaninoff H, Iung B, Donzeau-Gouge P, Chevreul K, Fajadet J, et al. Registry of transcatheter aortic-valve implantation in high-risk patients. $N$ Engl J Med. 2012;366:1705-15.

8. McVeigh ER, Guttman MA, Lederman RJ, Li M, Kocaturk O, Hunt T, et al. Real-time interactive MRI-guided cardiac surgery: aortic valve replacement using a direct apical approach. Magn Reson Med. 2006;56:958-64.
9. Horvath KA, Guttman M, Li M, Lederman RJ, Mazilu D, Kocaturk O, et al Beating heart aortic valve replacement using real-time MRI guidance Innovation. 2007;2:51-5.

10. Horvath KA, Mazilu D, Guttman M, Zetts A, Hunt T, Li M. Midterm results of transapical aortic valve replacement via real-time magnetic resonance imaging guidance. J Thorac Cardiovasc Surg. 2010;139:424-30.

11. Horvath KA, Mazilu D, Kocaturk O, Li M. Transapical aortic valve replacement under real-time magnetic resonance imaging guidance: experimental results with balloon-expandable and self-expanding stents. Eur J Cardiothorac Surg. 2011; 39:822-8.

12. Shrestha M, Folliguet T, Meuris B, Dibie A, Bara C, Herregods MC, et al. Sutureless Perceval S aortic valve replacement: a multicenter, prospective pilot trial. J Heart Valve Dis. 2009; 18:698-702.

13. Aguirre J, Waskowski R, Poddar K, Kapadia S, Krishnaswamy A, McCullough R, et al. Transcatheter aortic valve replacement: experience with the transapical approach, alternate access sites, and concomitant cardiac repairs. J Thorac Cardiovasc Surg. 2014;148:1417-22.

14. Mazilu D, Li M, Kocaturk O, Horvath KA. Self-expanding stent and delivery system for aortic valve replacement. J Med Device. 2012;6:410061-9.

15. Lederman RJ. Cardiovascular interventional magnetic resonance imaging Circulation. 2005;112:3009-17.

16. Al-Lamee R, Godino C, Colombo A. Transcatheter aortic valve implantation current principles of patient and technique selection and future perspectives Circ Cardiovasc Interv. 2011;4:387-95.

17. D’Onofrio A, Salizzoni S, Agrifoglio M, Lucchetti V, Musumeci F, Esposito G, et al. When does transapical aortic valve replacement become a futile procedure? An analysis from a national registry. J Thorac Cardiovasc Surg. 2014;148: 973-80.

18. Flameng W, Herregods MC, Vercalsteren M, Herijgers P, Bogaerts K, Meuris B. Prosthesis-patient mismatch predicts structural valve degeneration in bioprosthetic heart valves. Circulation. 2010;121:2123-9.

\title{
EDITORIAL COMMENTARY
}

\section{Do the "eyes" have it? Lobbying for magnetic resonance imaging to guide transcatheter aortic valve deployment}

\author{
Robert E. Michler, MD
}

See related article on pages 1067-72.

Of the many medical advances over the years, few have been as impactful on the practice of medicine as imaging technology. Initially used to peer into the human body for

From the Department of Surgery and the Department of Cardiothoracic and Vascular Surgery, Montefiore Medical Center, Albert Einstein College of Medicine, New York, NY.

Disclosures: Author has nothing to disclose with regard to commercial support.

Received for publication Feb 4, 2015; accepted for publication Feb 4, 2015.

Address for reprints: Robert E. Michler, MD, Montefiore Medical Center/Albert Einstein College of Medicine, Cardiothoracic and Vascular Surgery, 3400 Bainbridge Ave, MAP 5, New York, NY 10467 (E-mail: rmichler@montefiore.org).

J Thorac Cardiovasc Surg 2015;149:1072-3

$0022-5223 / \$ 36.00$

Copyright (c) 2015 by The American Association for Thoracic Surgery

http://dx.doi.org/10.1016/j.jtcvs.2015.02.024 diagnosis, with time imaging technology has become a tool in planning and monitoring medical and surgical treatments, aiding preoperative planning through 3-dimensional computer modeling, and real-time guidance during surgical interventions. There is probably no finer example of the merger of an imaging tool with a concurrent interventional therapy than percutaneous coronary intervention.

The success of transcatheter aortic valve replacement is exquisitely dependent upon imaging, and requires both fluoroscopy and echocardiography during the procedure to deploy a prosthetic aortic valve. In looking toward the future and thinking of improving deployment precision, Horvath and colleagues have been asking the question of whether the precision of valve deployment could be enhanced by using real-time magnetic resonance imaging (MRI) during the procedure. The motivation for this inquiry arises from the limitations of fluoroscopy and echocardiography relative to MRI. MRI is significantly superior at 